\title{
Morphological study of defects of the atrial septum within the oval fossa: implications for transcatheter closure of left-to-right shunt
}

\author{
Sheila M A G Ferreira, Siew Yen Ho, Robert H Anderson
}

\begin{abstract}
Objective-To determine the anatomical variability of the oval fossa in cases of atrial septal defect and to find out which factors might make such defects suitable or unsuitable for closure by umbrella or clamshell devices.

Design-100 specimens with defects of the atrial septum within the oval fossa were studied, especially the position of the defects within the fossa; the area of the defect in relation to the total area of the oval fossa; the shape of the rims and flap valve of the oval fossa; and the anatomical variability in the eustachian and thebesian valves.
\end{abstract}

Results-The oval fossa was displaced to the mouth of the inferior caval vein in four cases; displaced to the mouth of superior caval vein in two cases; placed on the middle of the interatrial wall in 43; or placed slightly towards the inferior caval vein in 51 . Because of their shape 29 of the hearts were considered to be unsuitable for transcatheter closure of the defect. This was because the defect was too large (16 hearts); the oval fossa was displaced to the mouth of the inferior caval vein (four hearts) or to the superior caval vein (two hearts); lacked its anterior rim (two hearts); lacked the posteroinferior rim (one heart); because a thick eustachian valve was displaced posteriorly forming a false posteroinferior rim (one heart); or because the strand of insertion of the floor of the oval fossa was too distant from the left atrial aspect (three hearts).

Conclusion- 68 hearts appeared to be ideal candidates for transcatheter closure; 3 would probably have been suitable; but 29 were unsuitable. These morphological variations might explain why the procedure has been unsuccessful in so many cases. Patients should be screened before any attempt is made at transcatheter closure.

In the past three decades there have been many advances in the use of interventional techniques in the treatment of congenital malformations of the heart including those of the atrial septum. ${ }^{1}$ Morphological studies show ${ }^{23}$ that not all communications that permit an interatrial shunt, and not even all those within the oval fossa, will be suitable for closure in this fashion. The refinements made in interventional cardiology, therefore, make it necessary to know the nature and extent of the morphological patterns of the oval fossa, and particularly of its deficiencies, which could interfere in closure by umbrella or clamshell devices. With this need in mind, we have investigated the variable anatomical patterns of deficiencies of the floor of the oval fossa, its muscular rims, and its relation to the other structures separating the cavities of the right and left atria. In this way, we hoped to identify difficulties that might occur during the introduction of catheters and the placement of the occluding device. We also hoped to identify the proportion of cases that might be unsuitable for non-surgical closure.

\section{Methods}

There are 352 specimens with deficiencies of the oval fossa in the cardiopathological collection of the Department of Paediatrics of the National Heart and Lung Institute of the University of London. From these we selected the first 100 specimens that were suitable for detailed analysis.

We selected hearts of any size or age in which the oval fossa had not been torn by Rashkind's procedure or repaired surgically. We also excluded any specimens in which associated cardiac malformations might have distorted the atrial septum, such as juxtaposition of the appendages, extreme hypoplasia of the left or right heart, criss-cross hearts, and so on. Specimens with other forms of interatrial communication (sinus venosus, ostium primum, and "coronary sinus" defects ${ }^{23}$ ) were also excluded.

We measured the longest and shortest diameters of each oval fossa. The radius of the circle was obtained by the formula $(X+Y) / 4$. Afterwards we estimated the size of the fossa by drawing the defect within the idealised circle of the fossa on graph paper. In this way, we calculated the proportion of the floor of the fossa that was deficient.

In all specimens we concentrated our descriptions on the right atrial aspect of the oval fossa. We looked at the position of the defect within the fossa, the position of the fossa on the walls between the atria, the extension of the defect towards the mouths of either the inferior 
or superior caval veins, the texture and the pattern of the flap valve of the fossa (the septum primum), the shape of the muscular rim, and the size and texture of the eustachian and thebesian valves.

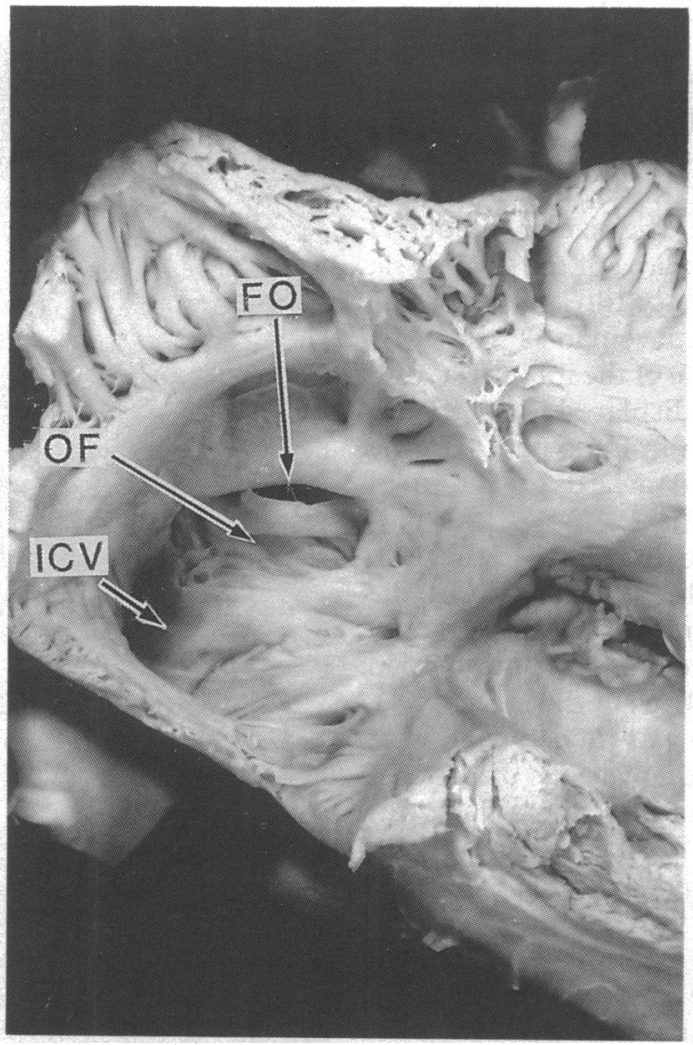

Figure 1 Specimen showing the oval fossa (OF) normally situated and slightly tilted towards the inferior caval vein (ICV) and a widely patent foramen ovale (FO).

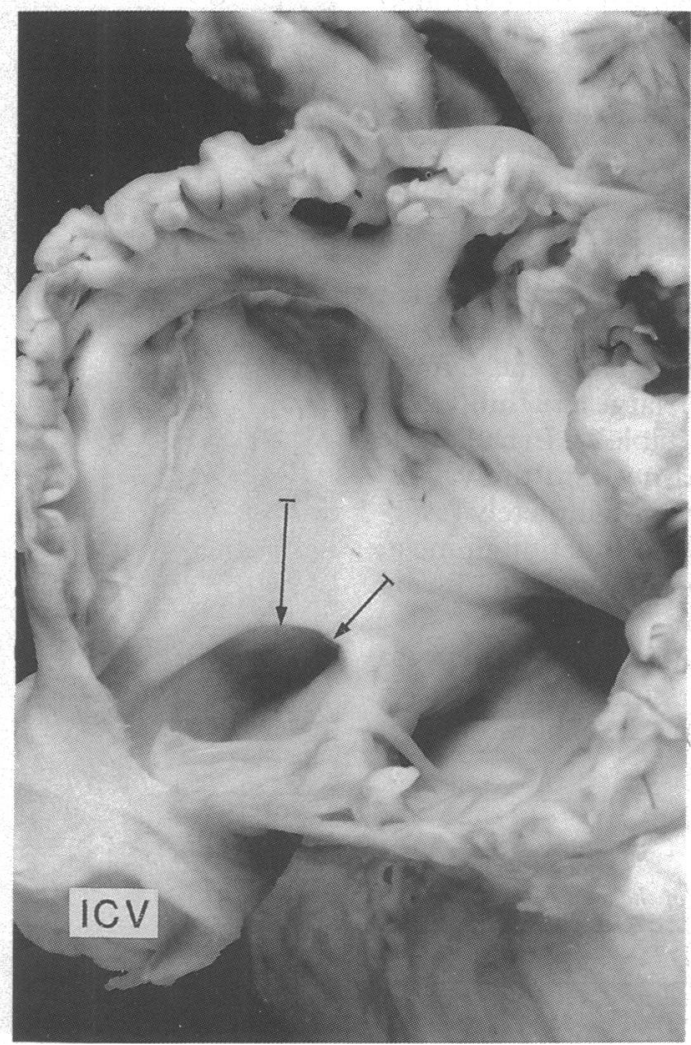

Figure 2 Specimen showing oval fossa displaced to the mouth of the inferior caval vein (ICV). Note the size of the anterosuperior and anteroinferior rims (arrows).
In each heart we then examined the left atrial aspect of the septum, noting any unusual muscle bands or undue proximity of the right pulmonary veins that might have made placement of a device inappropriate.

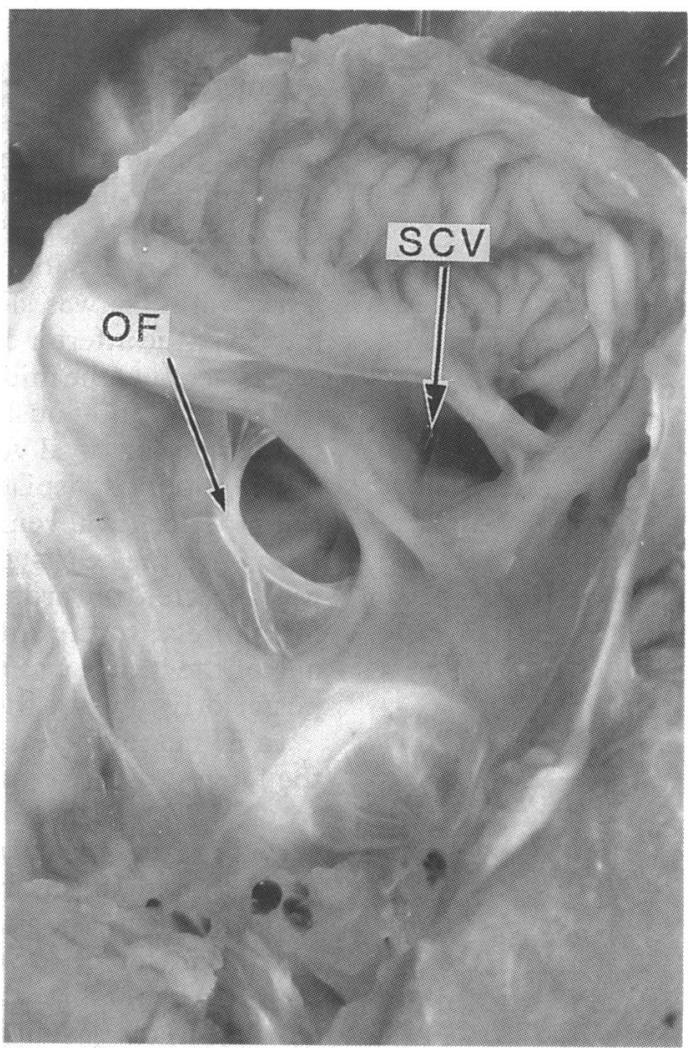

Figure 3 Specimen showing the oval fossa (OF)

displaced to the mouth of the superior caval vein (SCV).

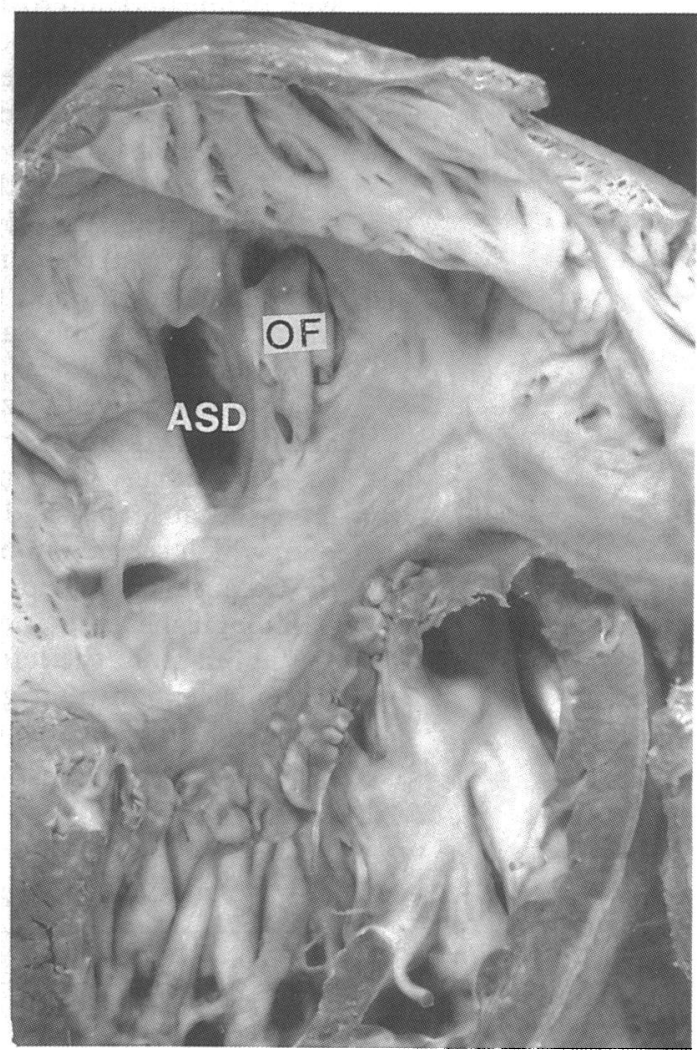

Figure 4 Specimen showing atrial septal defect (ASD) opened posteriorly of the oval fossa (OF) toward the posterior wall of the atria. 


\section{Results}

The youngest specimen examined came from a neonate of six hours, and the oldest was from a patient aged 35 years. There were 31 specimens from patients whose age was not known, but as far as could be judged from size most of them came from young children. Of the 100 hearts chosen for analysis, 17 had a patent oval foramen but no deficiency of the flap valve(fig 1 ). In 12 of the hearts the defect occupied $50 \%$ or more of the total area of the fossa. In four there was complete absence of the flap valve while, in the remaining 67 hearts, the size of the defect was less than $50 \%$ of the area of the oval fossa. The oval fossa was slightly tilted towards the mouth of the inferior caval vein in 51 hearts (fig 1). It occupied the middle of the interatrial wall in 43 but was obviously displaced to the mouth of the inferior caval vein in four (fig 2). In the other two it was displaced towards the mouth of the superior caval vein (fig 3 ).

In 99 of the hearts, the deficiency of the floor was directed towards the anterior rim of the fossa or else to the anteroinferior rim. In only one of the hearts was the defect found to open posteriorly towards the back of the atria (fig 4).

In 83 hearts the fossa was indeed oval, with the longest diameter taking various directions. In only 17 was the fossa round. Its floor was very thick and muscular in 48 , moderately thick in 27 , thin in 18 , showing a pattern of filigreed remnants in three, and virtually absent in the other four (table 1). Six hearts had fenestrated floors. In five of these the flap valve was very thin whereas in the remaining one it was thick.

The shape of the rims of the fossa also varied considerably. The rim was well delineated all around the fossa in 67 hearts. In contrast, it was well delineated anterosuperiorly but exhibited a flat posteroinferior border in 21 . In another eight of the hearts all the border was flat without any obvious rim. There was no anterior rim in two of the hearts (fig 5) and no posterior rim in one. In the final heart, the eustachian valve was so thick and posteriorly displaced that it formed a false posteroinferior rim (fig 6).

The eustachian and thebesian valves, when identified, could be categorised according to their texture, thickness, and size: namely, muscular (irrespective of size); large and thin; small and thin; or rudimentary (table 2 ). In half of the cases, however, we could not find the valves, either because they had been completely resorbed, which seemed to be the situation in most, or because they had been removed during pathological examination.

Viewed from the left atrial aspect, the fossa was adjacent to the right superior pulmonary veins in three hearts (fig 7). The strand of

Table 1 Patterns of the floor of the oval fossa

\begin{tabular}{lr}
\hline Pattern & No \\
\hline Very thick and muscular & 48 \\
Moderately thick & 27 \\
Thin & 18 \\
Filigreed remnant & 3 \\
Absent & 4 \\
\hline
\end{tabular}

insertion of the flap valve was far from the limits of the fossa in another three hearts (fig 8).

Our observations indicated that undue difficulties might have been encountered in closing the defects by means of a device in 29 hearts. In 16 hearts this was because the defect was too

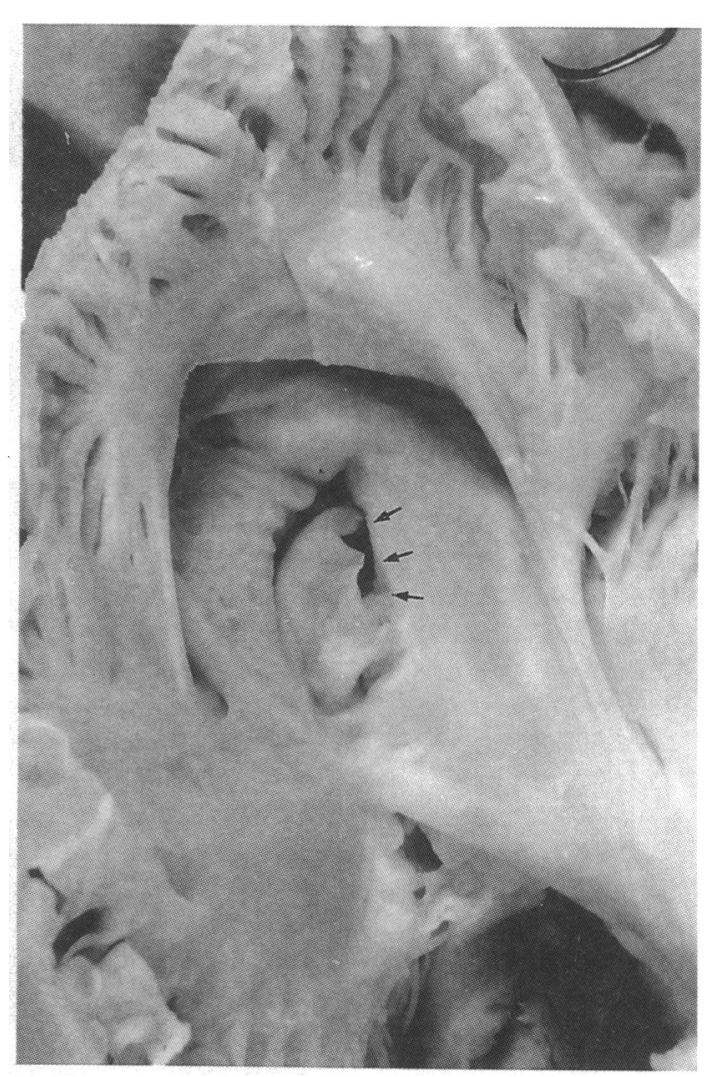

Figure 5 Specimen showing the oval fossa in which there is no anterior rim to anchor a device (arrows).
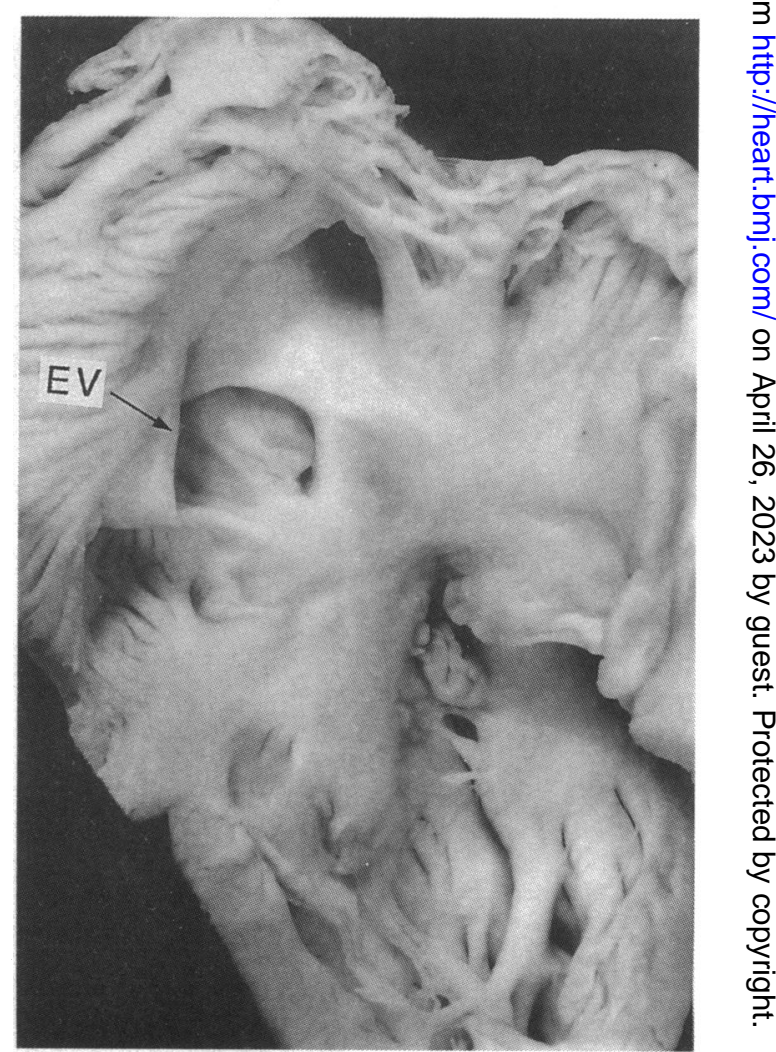

Figure 6 Specimen showing the eustachian valve (EV) that was unusually thick and posteriorly displaced to form a false posteroinferior rim to the oval fossa. 
large, either as a consequence of complete lack of development of the flap valve or because the defect was larger than half the area of the oval fossa. In four hearts it was because of displacement of the fossa towards the mouth of the inferior caval vein (in three of these the oval fossa, on its left atrial aspect, was also adjacent to the right superior pulmonary veins), and

Table 2 Patterns of eustachian and thebesian valves

\begin{tabular}{lcc}
\hline Pattern & Eustachian & Thebesian \\
\hline Muscular (irrespective of size) & 6 & 2 \\
Large and thin & 19 & 15 \\
Small and thin & 10 & 10 \\
Rudimentary & 15 & 26 \\
Not seen & 50 & 47 \\
\hline
\end{tabular}

*Completely resorbed or removed during pathological examination.

Figure 7 Specimen showing the oval fossa viewed from the left atrial aspect adjacent to the right pulmonary veins (RPV).
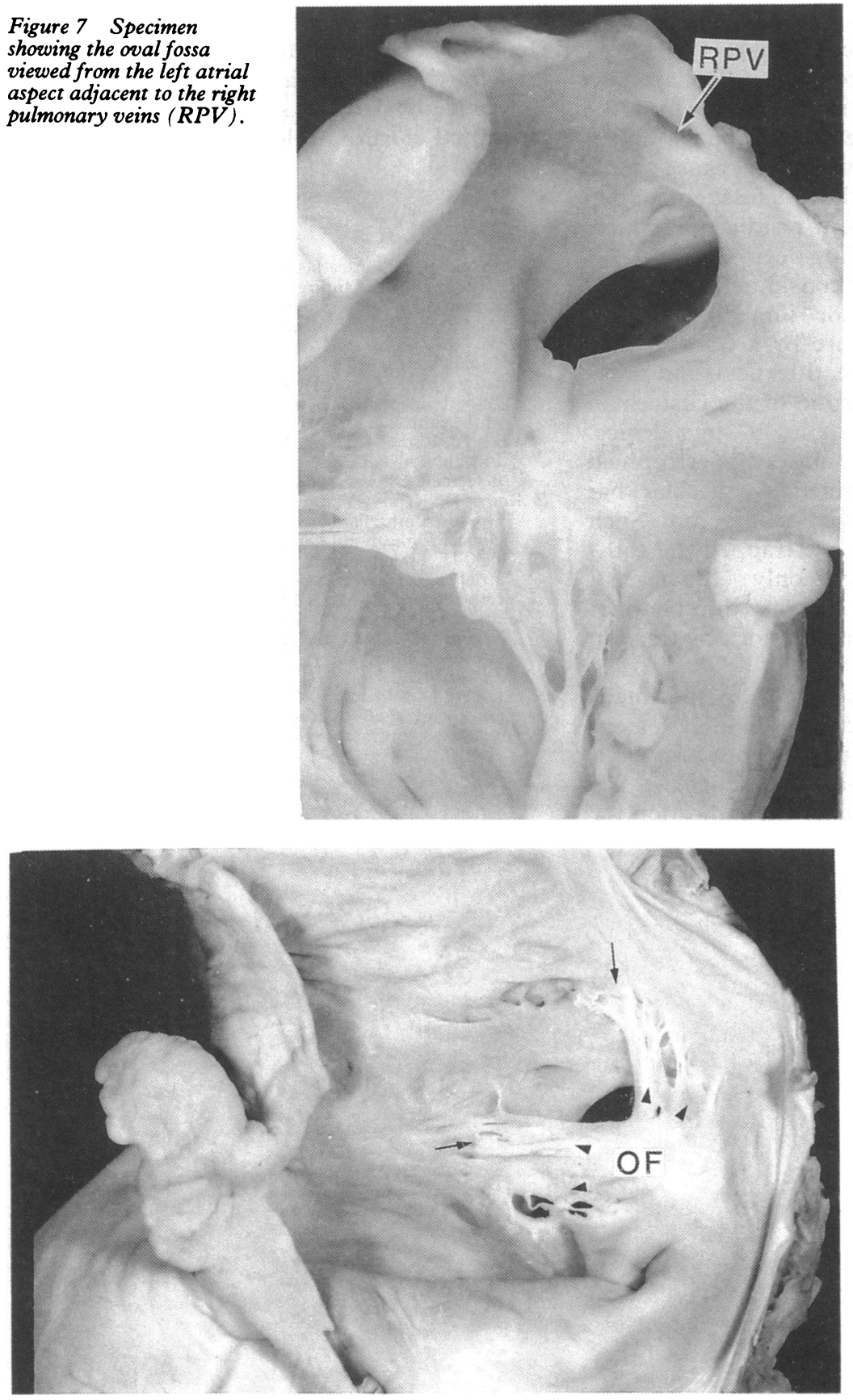

Figure 8 Specimen showing the oval fossa (OF) viewed from the left at:ial aspect showing the insertion of the flap valve (arrows) far from its usual attachment (arrow heads). because it extended towards the mouth of the superior caval vein in two. There was no anterior rim and no obvious facility to anchor the device in two hearts and no posteroinferior rim in one heart where the defect was situated adjacent to the posterior wall of the atria. The eustachian valve formed a false posteroinferior rim to the defect in one heart. In the three remaining hearts, the flap valve in the left atrium was inserted so far from the rim of the fossa that it could have allowed residual flow after attempted closure.

Other problems that could have caused technical difficulties in manoeuvring the sheath inside the right atrium were found in three hearts. There was hypertrophy of the terminal crest in two and presence of a large and muscular eustachian valve in the other. In one further heart a slit was discovered within the sinus septum which if undetected would have remained as an additional communication between the atria.

\section{Discussion}

Interventional cardiology has made it possible to close atrial septal defects in selected patients with devices inserted through catheters. ${ }^{1}$ A better understanding of the types of defects in the oval fossa that would be suitable for closure by this method, and those in which such attempted closure may be contra-indicated, depends on a full understanding of the anatomy of the oval fossa and the surrounding tissue. While others have examined the anatomy of the rim of the oval fossa and also its proximity to vital structures within the atria, ${ }^{45}$ the anatomical variability of these structures has rarely been considered. We carefully examined 100 postmortem specimens and noted the size and variability of deficiencies of the oval fossa in each.

From the right atrial aspect the atrial wall extends from the orifices of the superior and inferior caval veins to the attachment of the septal leaflet of the tricuspid valve. Within this extensive area only the oval fossa and its immediate rims separate the cavities of the two atrial chambers. Any hole outside this area is not, by definition, an atrial septal defect but rather an interatrial communication. ${ }^{2}$ By virtue of the more complex arrangement of such communications, it is only deficiencies within the oval fossa that, in the first instance, are likely to be suitable for closure by devices introduced on catheters. We have concentrated on defects in the oval fossa.

The results of our study suggest that some anatomical arrangements are not amenable to transcatheter repair. The location of the oval fossa was grossly abnormal in four specimensthat is, situated directly in front of the mouth of the inferior caval vein. Not only would this position make it difficult to distinguish the defect from an interatrial communication of the inferior sinus venosus type, but such holes would also be difficult to close without impeding flow from the inferior caval vein. In these cases, the posteroinferior rim may be too small to anchor the device satisfactorily without 
leaving a residual shunt. This was also true of two specimens in which the oval fossa was situated adjacent to the entrance of the superior caval vein.

Some studies have highlighted inadequacy of a muscular rim around the defect as a cause of embolisation of the device. ${ }^{15}$ We found five examples of this arrangement out of the 100 hearts we examined. Two lacked the anterior rim and one lacked the posterior rim, while the other two, where the defect was displaced to the mouth of the superior caval vein, lacked the superior rim.

Clinical experience has shown that the diameter of the device used for attempted closure must be 10 to $15 \mathrm{~mm}$ greater than the area of the septal deficiency, ${ }^{6}$ or at least 1.6 times the diameter of the defect as determined by sizing with balloons at catheterisation. ${ }^{5}$ The defect was equal to or bigger than half the area of the oval fossa in 16 of these hearts. This would rule out attempted closure.

There may also be problems if the flap valve on the left atrial aspect is inserted far from the rim of the fossa. Depending upon its extent such malinsertion could also cause some malalignment between the oval fossa and its flap valve, thereby allowing a residual flow after placement of the occluding device.

Apart from the major considerations discussed above, other minor impediments can prevent successful closure by a transcatheter technique. Thus the size, thickness, and proximity of the septal insertion of the eustachian valve may cause it to be "caught" by a device. In one heart we also found a hypertrophied terminal crest that formed a muscular bar across the right atrial cavity near its roof. Such an arrangement could have trapped the catheter sheath. More commonly (18 hearts), however, we noted that the very thin floors of the oval fossa could have been torn by traction on the sheath.

In this study we included 17 specimens where the oval foramen was widely patent because the primary septum was not fused, but the flap valve itself was not deficient. We included these because later the indication for transcatheter closure may be broadened. It could be used in some particular groups of patients who late in life have unsaturation at the atrial level because of increased pressure in the right side of the heart, patients who have percutaneous dilatation of the mitral valve, a procedure that leaves a shunt ${ }^{78}$ at the atrial level, and the large number of young patients with a patent oval foramen in whom stroke may be caused by paradoxical embolism. ${ }^{9}$

Only 68 of the 100 hearts we examined appeared to be ideal for transcatheter closure. Three hearts would probably have been suitable. In 29, however, we found anatomical impediments that would have countervailed against successful closure. It is always unwise to predict the impossibility of future closure on the basis of study of necropsied hearts, as shown by the apparent success of balloon dilatation of native coarctation despite histological studies pointing to its inadvisability. Our study does indicate, none the less, that, at least initially, great care should be exercised and only ideal cases should be referred for attempted closure of defects of the atrial septum.

1 Rocchini AP. Transcatheter closure of atrial septal defects:

past, present and future. Circulation 1990;82:1044-5.
2 Beerman LB, Zuberbuhler FR. Atrial septal defect. In: Anderson RH, Macartney FJ, Shinebourne EA, Tynan M, eds. Paediatric cardiology. Edinburgh: Churchill Livingstone, 1989:541-62.

3 Ettedgui JA, Siewers RD, Anderson RH, Park SC, Pahl E, Zuberbuhler JR. Diagnostic echocardiographic features Zuberbuhler JR. Diagnostic echocardiographic feature

4 Lock JE, Rome JJ, Davis R, Van Praagh S, Perry SB, Van Praagh R, et al. Transcatheter closure of atrial septa defect: experimental studies. Circulation 1989;79:1091-9.

5 Rome JJ, Keane JF, Stanton BP, Spevak PJ, Lock JE Double-Umbrella closure of atrial septal defects: initia clinical applications. Circulation 1990;82:751-8.

6 King TD, Thompson SL, Steiner C, Mills NL. Secundum atrial septal defect: nonoperative closure during cardiac catheterization. JAMA 1976;235:2507-9.

7 L'Epine Y, Drobinski G, Sotirov Y, Moussallem N, Voudris $\mathrm{V}$, Komajda $\mathbf{M}$, et al. Right heart failure due to an interatrial shunt after mitral balloon dilatation. Eur Heart J 1989;10:285-7.

8 Goldberg N, Roman CF, Cha SD, Weiner R, Maranhao V, Elderdge J, et al. Right to left interatrial shunting following balloon mitral valvuloplasty. Cathet Cardiovasc Diagn 1989;16:133-5.

9 Lechat P, Mas JL, Lascault G, Loron P, Theard M, Klimczac M, et al. Prevalence of patent foramen ovale in patients with stroke. N Engl J Med 1988;318:1148-52. 